\title{
REVISIONES
}

\section{Atributos de una docencia de calidad en la educación superior: una revisión sistemática*}

\author{
Attributes of quality teaching in higher education: a systematic review
}

\author{
Óscar Jerez Yáñez, ${ }^{a}$ César Orsini Sánchez, ${ }^{a b}$ Beatriz Hasbún Heldac \\ ${ }^{a}$ Centro de Enseñanza y Aprendizaje de la Facultad de Economía y Negocios de la Universidad de Chile \\ Telf.: (56) 229772030. Correo electrónico: ojerez@fen.uchile.cl \\ ${ }^{\mathrm{b}}$ Correo electrónico: corsini@fen.uchile.cl \\ ${ }^{\mathrm{c} C}$ Correo electrónico: bhasbun@fen.uchile.cl
}

\begin{abstract}
RESUMEN
La presente revisión analiza de forma sistemática los atributos de una docencia de calidad en la educación superior, extrayendo información sobre características y cualidades que constituyen una docencia de 'excelencia'. La búsqueda documental se llevó a cabo a través de la triangulación de diversas fuentes, identificando 25 artículos. Mediante un análisis temático, dos autores de forma independiente analizaron y codificaron los atributos de un docente de calidad, clasificándolos en tres competencias: las genéricas (características personales, actitudinales y comunicativas); las pedagógicas (estrategias de enseñanza-aprendizaje y de planificación-gestión); y las disciplinares. Se presentan sus implicancias y sus indicadores específicos. Las características identificadas son factibles de ser modificadas, aprendidas y entrenadas. Estudiantes y profesores resaltan la necesidad fundamental de prepararse para ser profesor universitario, teniendo presente el papel crucial que juegan las variables de tipo genéricas, pedagógicas y disciplinares, tanto en la interacción profesor-estudiante como en la facilitación del aprendizaje.
\end{abstract}

Palabras clave: competencias docentes, docencia efectiva, educación superior, habilidades docentes, revisión de literatura.

\section{ABSTRACT}

The aim of this systematic review is to analyse the attributes that constitute high quality teaching in higher education by extracting data on the characteristics of 'excellent' teachers. A literature search was conducted through the triangulation of multiple sources, identifying 25 articles. Through a thematic analysis, two authors independently reviewed and coded the attributes of quality teaching classifying them into three categories: generic competencies (personal, attitudinal, and communicational characteristics); pedagogical competencies (teaching and learning strategies, and planning and management); and disciplinary competencies. Further implications of each attribute with their specific indicators are described. The characteristics identified, in general, are feasible to be trained, modified, and learned. Students and faculty emphasised the critical need for university teachers to be professionally prepared to teach and for them to consider the relevance of generic, pedagogical, and disciplinary variables as determinants of a good teacher-student interaction and for the facilitation of learning.

Key words: teacher competencies, teacher effectiveness, higher education, teaching skills, literature reviews.

El presente artículo se enmarca en el Proyecto FONDECYT de Iniciación no 11150794 denominado "Qué hacen los mejores profesores universitarios en clases masivas: Un estudio de casos múltiples". 


\section{INTRODUCCIÓN}

La calidad de la docencia universitaria se encuentra estrechamente relacionada con la calidad de los aprendizajes de los estudiantes y, por tanto, con la formación de profesionales. Es por ello que a nivel mundial se observa una mayor atención sobre la calidad de la enseñanza y el aprendizaje universitarios, así como una creciente presión para garantizar y generar evidencias de una enseñanza eficaz (Devlin y Samarawickrema, 2010; Glenn et al., 2012). Lo anterior se ha traducido en la incorporación de la calidad de la docencia como un factor relevante en diversos estándares de acreditación, tanto a nivel de programas de estudio como de instituciones (AACSB, 2015; AMBA, 2015).

Durante laúltima década, la educación superior hasido objetode variadas investigaciones, informes y reportes, los cuales convergen en determinar el tema de la calidad como uno de los principales desafíos del sistema de educación terciara (Espinoza y González, 2013; Gambi y González, 2013; Katz y Spence, 2009). Algunas de las causas explicativas del actual problema de la calidad en educación superior son su masificación en las últimas dos décadas como resultado de la multiplicación y diversificación de las instituciones, las restricciones provenientes del gasto público y la necesidad de perfeccionar un sistema de aseguramiento de la calidad (OECD, 2013). Sin embargo, la discusión actual sobre calidad de la educación superior ha olvidado o ignorado otro importante factor: los profesores universitarios y su práctica docente. Este último factor adquiere sentido teniendo en cuenta la privilegiada responsabilidad del docente en los procesos de enseñanza y aprendizaje, por lo que su rol es altamente relevante en la calidad de la formación profesional y académica de las actuales y futuras generaciones (Küster-Boluda y Vila López, 2012).

Este hecho se hace evidente en diferentes contextos y latitudes. Young y Shaw (2014) plantean que la labor del docente en la universidad es compleja, y por tanto definir qué se entiende por calidad es aún más complejo, considerando que se trata de un concepto controvertido. Para Bain, una docencia de calidad es aquella capaz de provocar un aprendizaje extraordinario en los estudiantes, es decir, un desarrollo intelectual y personal permanente en el tiempo (Bain, 2004). Incluso en las carreras de la salud se vinculan el axioma docencia eficaz con la calidad en la atención clínica que brindarán sus futuros profesionales (Van Der Leeuw et al., 2013).

En Europa, con la irrupción del Espacio Europeo de Educación Superior, se posicionó a la docencia como elemento fundamental para asegurar la calidad: "los docentes son el recurso de aprendizaje más importante a disposición de la mayoría de los estudiantes" (ENQA, 2005, p. 18). La garantía por la calidad de la docencia en las universidades europeas se ha vuelto un requerimiento en los procesos de gestión (Pozo et al., 2011). En EEUU, la relevancia de este factor se ha vuelto un preponderante irrenunciable de la acción formativa actual (Bain, 2004; Fry, Ketteridge y Marshall, 2008; Marzano, 2010). En Australia, las principales preocupaciones en estos años han sido la mejora de la enseñanza y el desarrollo profesional de los profesores; así, la docencia se ha convertido en el factor más importante de la calidad y en un actor central del trabajo de las universidades (Lee, Manathunga y Kandlbinder, 2010). En Chile, por ejemplo, esto cobra aún más relevancia si tomamos en cuenta que desde el año 2007 a la fecha, el $42 \%$ de los estudiantes que ingresan a las universidades tradicionales constituyen primera generación en acceder a la educación superior dentro de su grupo familiar (PUCV, 2013), lo que desafía la visión tradicional del docente y lo moviliza hacia nuevos escenarios de actuación profesional. 
Sin embargo, la investigación sobre la práctica docente de profesores universitarios es una tendencia relativamente reciente (Hannah, Stewart y Thomas, 2011). El conocer los atributos y características de las prácticas docentes reconocidas como de excelencia en los diferentes contextos y realidades en los que se inserta la educación superior permitirá orientar con mayor pertinencia el desarrollo de la docencia universitaria, especialmente en países en vías de desarrollo.

No obstante, hablar de los atributos de una docencia universitaria de calidad no es tarea fácil ni trivial. A este nivel cabe preguntarse, ¿en qué consiste esa excelencia o calidad de la docencia de un profesor universitario?, ¿qué acciones realizan ciertos docentes para conseguir una importante y duradera influencia en el desarrollo intelectual y personal de los estudiantes?, ¿cuáles son los atributos que la comunidad universitaria valora con mayor fuerza en un docente de calidad?, ¿qué estudios han abordado el tema?, ¿qué enfoques han utilizado?, ¿cuáles son las principales conclusiones? (Bain, 2004; Casero, 2010; Sánchez, 2013).

De esta forma, la presente revisión tiene por objetivo analizar y describir de forma sistemática los atributos de una docencia de calidad en la educación superior, a través de la revisión de estudios empíricos que involucran tanto a estudiantes como docentes, y publicados en idioma castellano e inglés desde el año 2000. El objetivo es extraer información sobre las características y cualidades que constituyen a un docente de 'excelencia' en el ámbito de la educación superior.

\section{MÉTODO}

La búsqueda de información se llevó a cabo entre abril y mayo 2015, siguiendo la guía propuesta por la declaración PRISMA para el reporte de revisiones sistemáticas y metaanálisis (Moher et al., 2009). Los criterios de inclusión exclusión aplicados se encuentran especificados en la Tabla 1. Se llevó cabo una búsqueda preliminar con el fin de explorar la evidencia existente, refinar el algoritmo de palabras claves, y con el objetivo de identificar revisiones similares realizadas por otros autores. Esta búsqueda fue efectuada en las bases de datos ERIC y en PsycINFO (accedidas a través de EBSCO), utilizando la siguiente combinación de palabras claves: (Quality OR Attributes OR Characteristics OR Excellence) AND (Teachers OR Tutors OR Faculty) AND (Higher Education OR University OR Tertiary Education). Como resultado de esta inmersión inicial se incorporaron palabras claves adicionales para aumentar la sensibilidad de la búsqueda y, con base en esos resultados, se decidió expandir la búsqueda a otras bases de datos y sitios web de revistas de relevancia para así aumentar la especificidad de la revisión. 
Tabla 1. Criterios de inclusión y exclusión aplicados a la búsqueda de literatura

\begin{tabular}{|c|c|}
\hline Criterios de Inclusión & Criterios de Exclusión \\
\hline $\begin{array}{l}\text { 1. Estudios empíricos o primarios enfocados } \\
\text { en los atributos de una docencia de calidad } \\
\text { derivados de las prácticas docentes o de la } \\
\text { percepción de estudiantes y/o profesores }\end{array}$ & $\begin{array}{l}\text { 1. Estudios no empíricos o secundarios, tales } \\
\text { como revisiones, editoriales, comentarios } \\
\text { y libros }\end{array}$ \\
\hline $\begin{array}{l}\text { 2. Estudios que reporten investigación en } \\
\text { Educación Superior }\end{array}$ & $\begin{array}{l}\text { 2. Estudios en poblaciones diferentes a estu- } \\
\text { diantes o profesores en educación superior }\end{array}$ \\
\hline $\begin{array}{l}\text { 3. Estudios cuantitativos con adecuadas } \\
\text { definiciones, operacionalización de } \\
\text { conceptos y análisis de datos }\end{array}$ & $\begin{array}{l}\text { 3. Estudios publicados en idiomas diferentes } \\
\text { al Castellano o Inglés }\end{array}$ \\
\hline $\begin{array}{l}\text { 4. Estudios cualitativos con adecuadas } \\
\text { definiciones de conceptos, métodos } \\
\text { confiables, análisis de datos y } \\
\text { conclusiones creíbles y válidas }\end{array}$ & 4. Estudios publicados antes del año 2000. \\
\hline $\begin{array}{l}\text { 5. Estudios disponibles en idioma Castellano } \\
\text { e Inglés }\end{array}$ & $\begin{array}{l}\text { 5. Estudios enfocados en la validación y/o } \\
\text { cons-trucción de instrumentos }\end{array}$ \\
\hline $\begin{array}{l}\text { 6. Estudios publicados entre los años } 2000 \\
\text { y } 2015\end{array}$ & \\
\hline
\end{tabular}

La búsqueda electrónica final se llevó a cabo a través de cinco bases de datos (ERIC, Medline, PsycINFO, Web of Science y Scopus) y en los sitios web de revistas relevantes. De forma adicional se buscó en la literatura gris (Open Grey) y en las citas bibliográficas de artículos seleccionados.

El algoritmo final de palabras claves se realizó con base en los conceptos de 'Quality AND Teachers AND Higher Education'. Cada concepto fue expandido utilizando sinónimos y adaptado a cada tesauro de las distintas bases de datos (Haig y Dozier, 2003). La Tabla 2 muestra la combinación de palabras claves utilizadas en la base de datos ERIC. Se fijó, a priori, un periodo de 15 años para así generar una revisión actualizada de la información.

Dos autores revisaron de forma independiente los resúmenes de cada estudio seleccionado, aplicando los criterios de inclusión exclusión previo a la revisión a texto completo. Frente a discrepancias de criterio se llegó a consenso a través de la mediación de un tercer autor. La Figura 1 presenta un resumen del proceso de búsqueda y revisión.

Se utilizó el programa de análisis cualitativo Nvivo® (QSR International, Doncaster, Australia) para extraer, codificar y analizar la metodología y resultados de los estudios seleccionados. Para guiar y evaluar la calidad de las metodologías aplicadas, tanto cualitativas como cuantitativas, se utilizaron las listas del 'Critical Appraisal Skills Programme' (Singh, 2013). 
Tabla 2. Identificación y expansión de los conceptos base de búsqueda: algoritmo aplicado en la base datos ERIC (accedida a través de EBSCO, 30-04-2015)

\begin{tabular}{|l|l|}
\hline \multicolumn{2}{|l|}{ Búsqueda 1: expansión de concepto 'Quality' (palabras combinadas con OR) } \\
\hline & $\begin{array}{l}\text { Tesauro: Teacher Characteristics - Teaching Styles- Diversity (Faculty) - Teacher } \\
\text { Attitudes - Teacher Behavior - Teaching Skills - Excellence in Education - Teacher } \\
\text { Effectiveness }\end{array}$ \\
\hline & Búsqueda libre: quality - attribut* \\
\hline Búsqueda 2: expansión de concepto 'Teachers' (palabras combinadas con OR) \\
\hline & $\begin{array}{l}\text { Teasuro: Teachers - Tutors - Faculty - College Faculty - Part Time Faculty - Women } \\
\text { Faculty }\end{array}$ \\
\hline Búsqueda 3: expansión de concepto 'Higher Education' (palabras combinadas con OR) \\
\hline $\begin{array}{l}\text { Tesauro: Higher Education - Postsecondary Education - Undergraduate Study - } \\
\text { Universities }\end{array}$ \\
\hline Búsqueda libre: University \\
\hline $\begin{array}{l}\text { Búsqueda 4: búsquedas 1, } 2 \text { y } 3 \text { combinadas con AND y con los delimitadores de texto completo, } \\
\text { entre año 2000 y 2015, artículos de revistas, educación postsecundaria, idioma castellano e inglés. }\end{array}$ \\
\hline
\end{tabular}

Para extraer la información referida a los resultados se realizó un análisis temático de codificación abierta, codificación central y, finalmente, una fase de interpretación y reflexión de la información generada (Hernández, Fernández y Baptista, 2010). Con todos los datos emergidos se crearon tablas de resumen de métodos y resultados relevantes de los artículos seleccionados. Dos autores analizaron de forma independiente los estudios, siguiendo las fases antes descritas, para posteriormente reunirse, comparar resultados y llegar a un acuerdo para el reporte final. 
Figura 1. Diagrama de flujo resumen del proceso de búsqueda y revisión

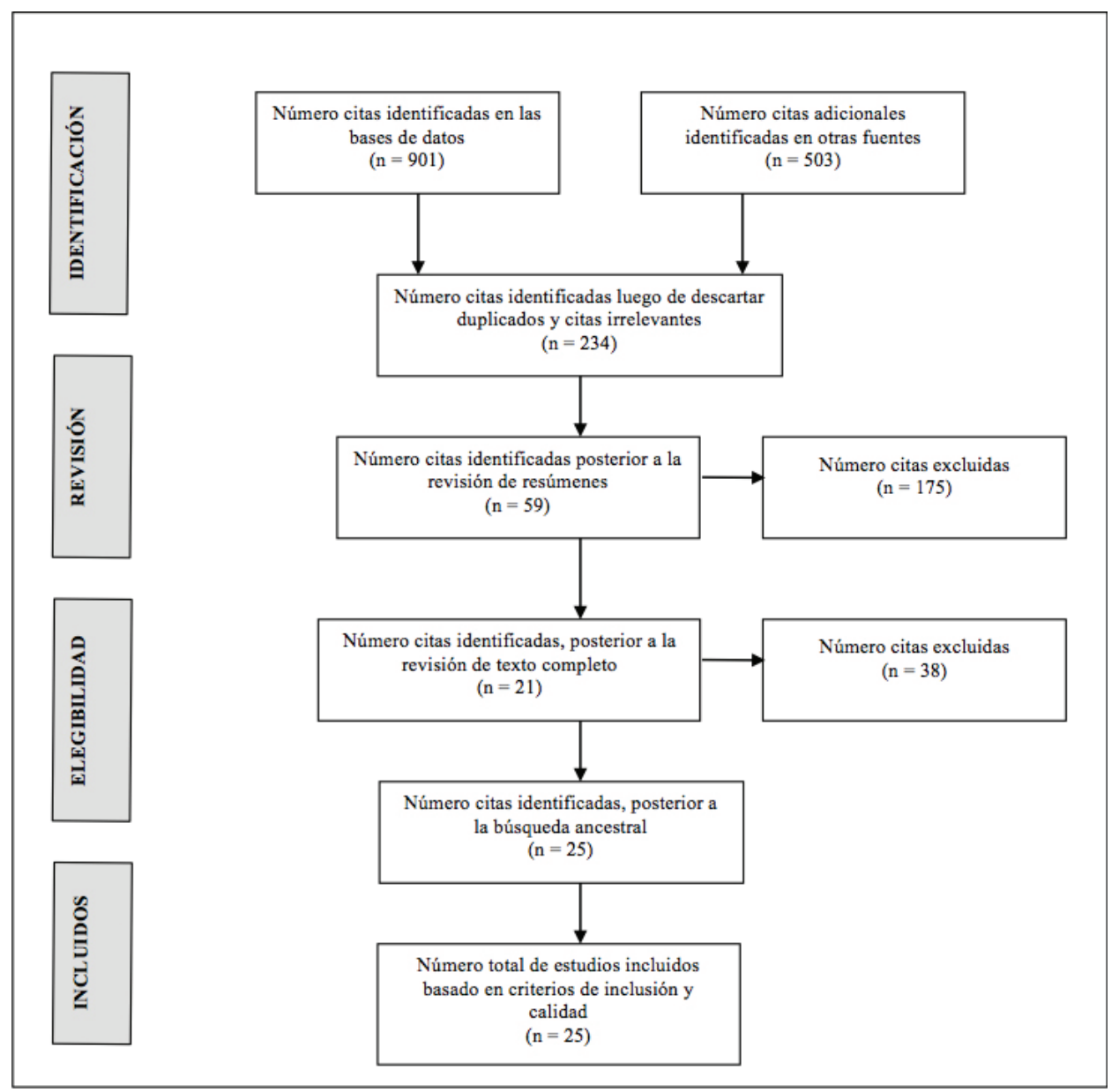

\section{RESULTADOS}

Se obtuvieron 1404 referencias, de la cuales se mantuvieron 234 luego de la eliminación de duplicados y referencias irrelevantes. Luego de la revisión de resúmenes, se redujo el número a 59 artículos y luego, posterior a la revisión a texto completo, a 21 (Figura 1). Las principales razones por la cuales se excluyeron artículos fue por corresponder a investigaciones secundarias, estar enfocados en educación primaria o secundaria, y por no referirse específicamente al tema de estudio. Se incorporaron 4 artículos luego de la búsqueda de referencias bibliográficas, quedando así en un total de 25 artículos, de los cuales todos fueron considerados de calidad (Singh, 2013). El resumen de métodos y resultados de cada estudio se presentan en la Tabla 3.

Los estudios seleccionados provienen de una gran variedad de latitudes, reflejando así la relevancia del tema a nivel global. Es posible afirmar que existen dos grandes tipos de 
estudios. El primer tipo se relaciona con la percepción de los estudiantes y profesores sobre los atributos de una docencia de excelencia (Alweshahi, Harley y Cook, 2007; Bartram y Bailey, 2009; Basow, Phelan y Capotosto, 2006; Bhattacharya, 2004; Cabalín y Navarro, 2010; Casero, 2010; Davies, Leung y Dunne, 2012; Duvivier et al., 2009; Friz, Sanhueza y Figueroa, 2011; Gargallo et al., 2010; Glenn et al., 2012; Jahangiri et al., 2013; Marín et al., 2011; Martínez, García y Quintanal, 2006; Parpala et al., 2011; Singh et al., 2013; Torok, McMorris y Lin, 2010); y el segundo tipo se vincula a la comprensión y caracterización de las prácticas de profesores reconocidos por su comunidad como de excelencia (Cid, Pérez y Zabalza, 2013; Cox y Swanson, 2002; Davidovitch y Milgram, 2006; De la Rosa, 2005; Gargallo, 2008; Hativa, Barak y Simhi, 2001; Monereo y Domínguez, 2014; Vajoczki et al., 2011). Este último grupo de estudios, aunque en minoría, refleja una mayor profundización en la comprensión de este hecho.

La interrogante sobre qué componentes constituyen una docencia de calidad ha sido estudiada mayoritariamente a través de métodos cuantitativos de corte transversal, cualitativos fenomenológicos y mixtos, involucrando a distintos sujetos en distintos contextos expresando visiones similares. El acceso a la información y la recolección de datos se llevó a cabo principalmente mediante tres vías: el acceso mediante el discurso, mediante respuesta a reactivos y a través de la observación de comportamientos (Cid et al., 2013).

A través del análisis temático efectuado, podemos clasificar los atributos de una docencia de calidad en tres grandes temas o competencias. El primero corresponde a las competencias genéricas (Peso relativo 37,73\%), subdivididas en características personales, actitudinales y comunicativas. El segundo corresponde a las competencias pedagógicas (Peso relativo 47,56\%), subdivididas en estrategias de enseñanza-aprendizaje y de planificación-gestión. La tercera temática corresponde a las competencias de tipo disciplinares (Peso relativo 14,71\%). Las tres temáticas con los subtemas e indicadores correspondientes han sido resumidas y se presentan en las tablas 4,5 y 6 . A continuación se presenta un análisis en mayor profundidad en relación a cada una de estas competencias. 
Estudios Pedagógicos XLII, Nº 3: 483-506, 2016

ATRIBUTOS DE UNA DOCENCIA DE CALIDAD EN LA EDUCACIÓN SUPERIOR: UNA REVISIÓN SISTEMÁTICA

\begin{tabular}{|c|c|c|c|c|c|}
\hline 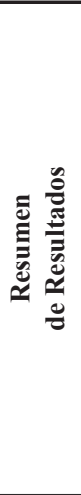 & 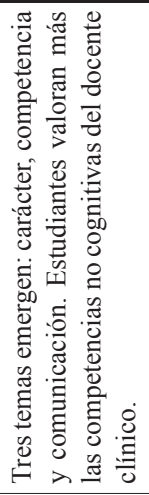 & 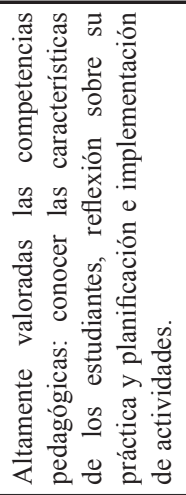 & 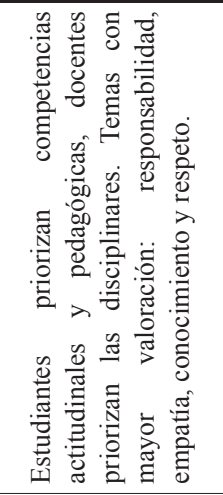 & 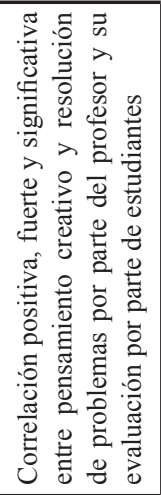 & 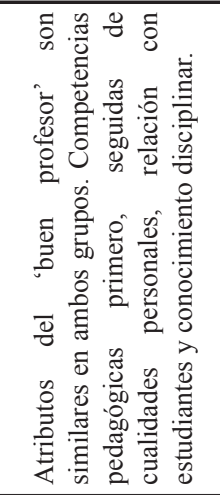 \\
\hline 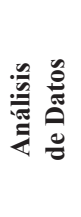 & 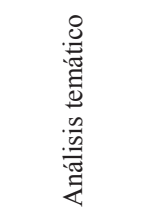 & 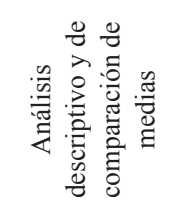 & 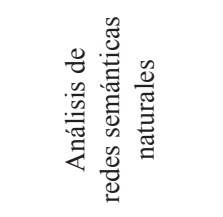 & 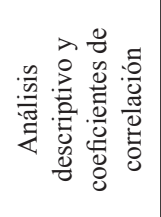 & 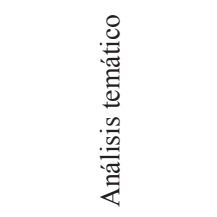 \\
\hline 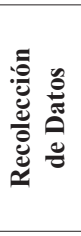 & 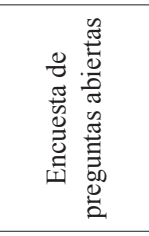 & 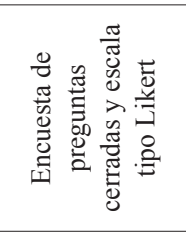 & 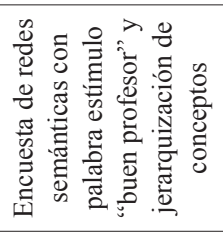 & 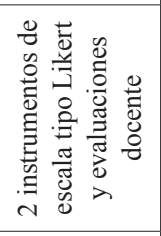 & 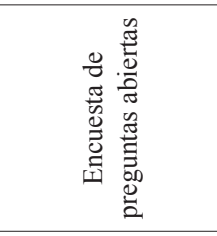 \\
\hline 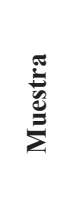 & 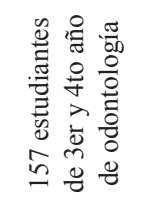 & 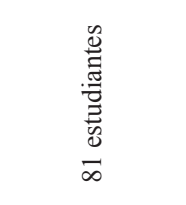 & 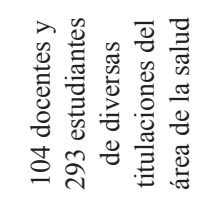 & 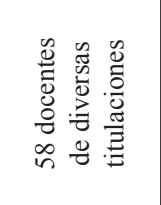 & 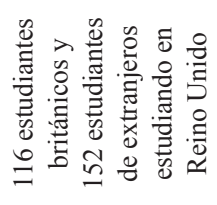 \\
\hline 总总 & 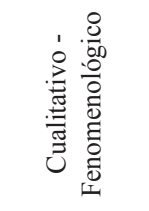 & 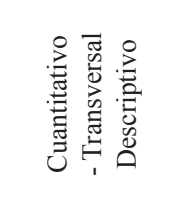 & 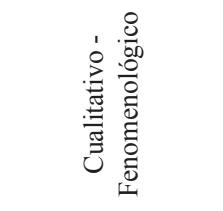 & 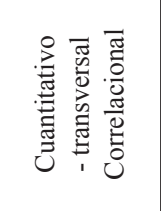 & 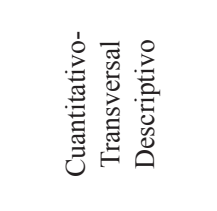 \\
\hline 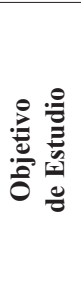 & 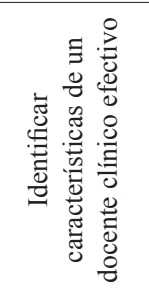 & 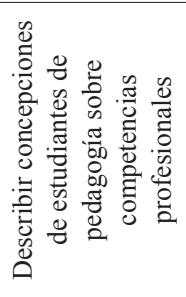 & 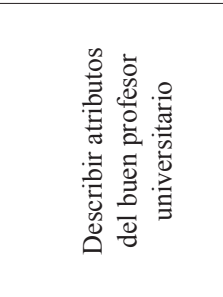 & 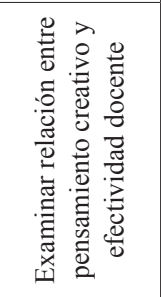 & 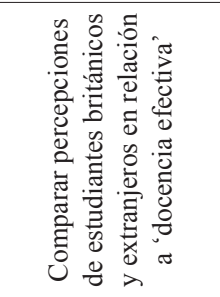 \\
\hline 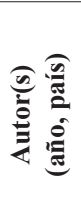 & 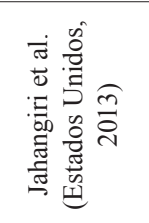 & 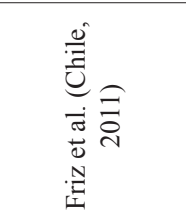 & 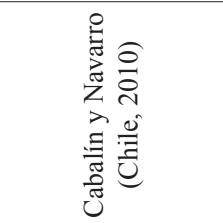 & 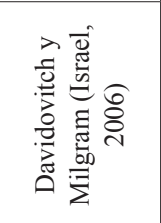 & 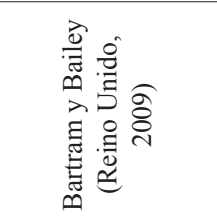 \\
\hline
\end{tabular}


Estudios Pedagógicos XLII, Nº 3: 483-506, 2016 ATRIBUTOS DE UNA DOCENCIA DE CALIDAD EN LA EDUCACIÓN SUPERIOR: UNA REVISIÓN SISTEMÁTICA

\begin{tabular}{|c|c|c|c|c|}
\hline 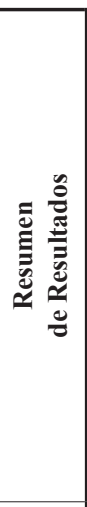 & 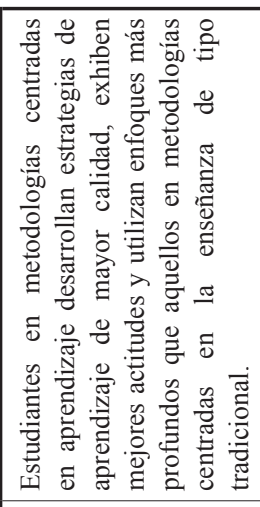 & 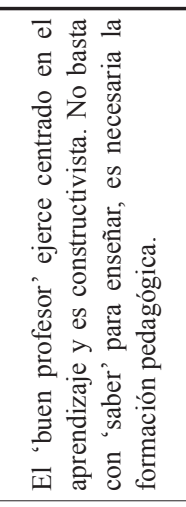 & 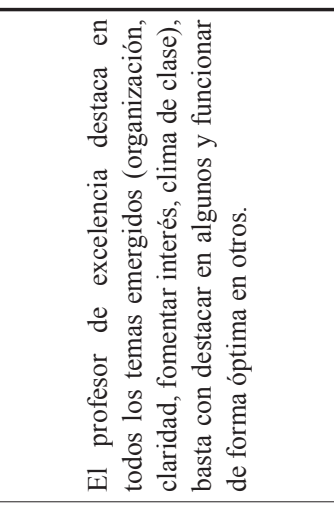 & 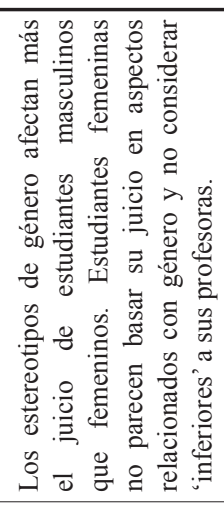 \\
\hline 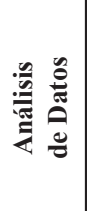 & 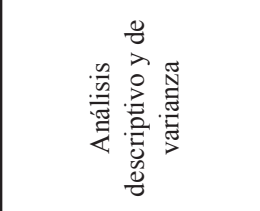 & 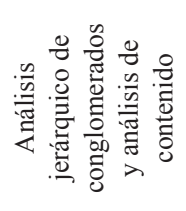 & 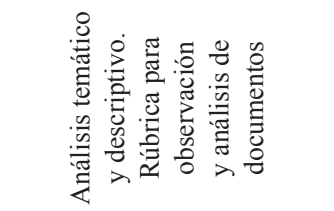 & 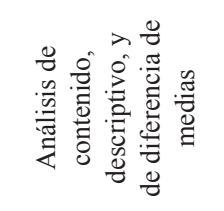 \\
\hline 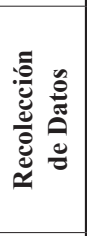 & 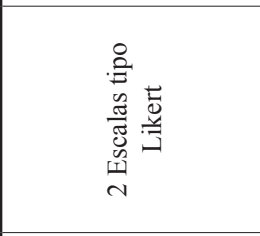 & 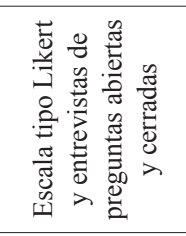 & 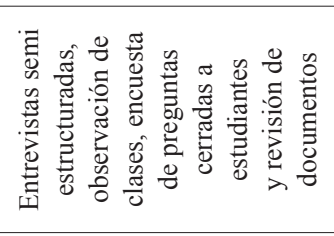 & 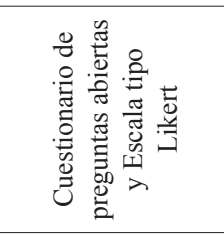 \\
\hline 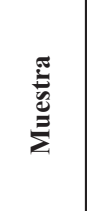 & 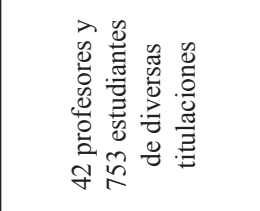 & 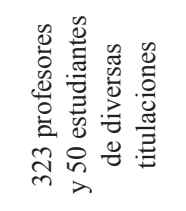 & 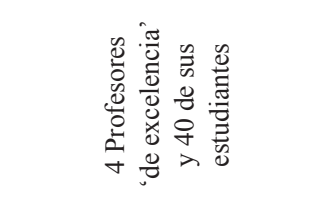 & 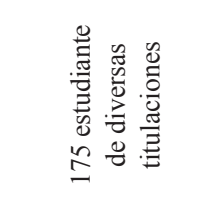 \\
\hline 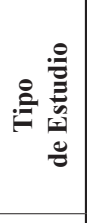 & 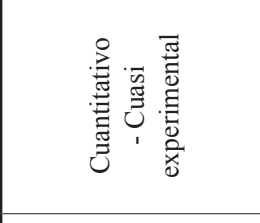 & 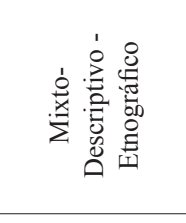 & 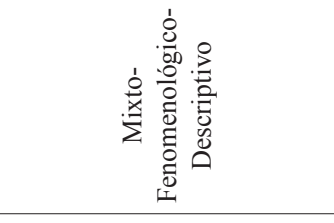 & 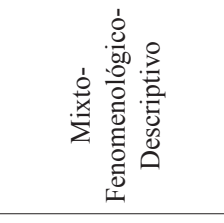 \\
\hline 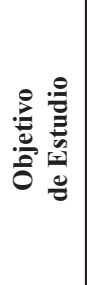 & 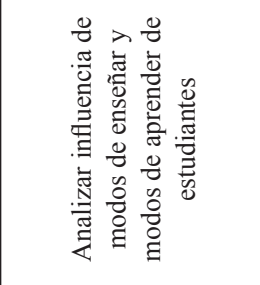 & 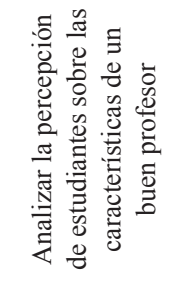 & 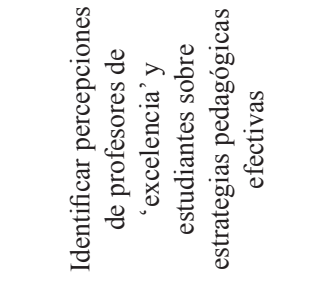 & 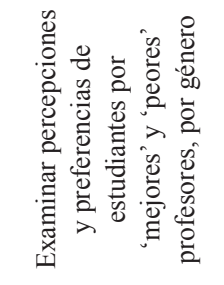 \\
\hline 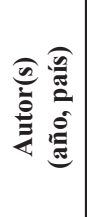 & 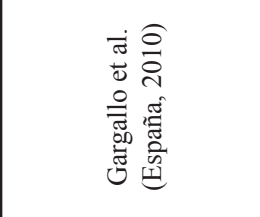 & 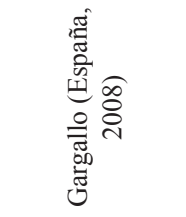 & 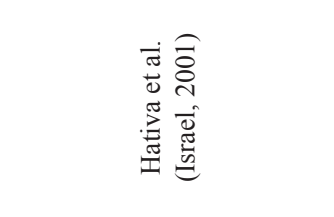 & 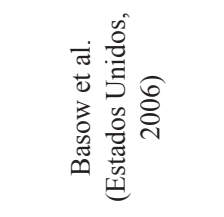 \\
\hline
\end{tabular}


Estudios Pedagógicos XLII, Nº 3: 483-506, 2016

ATRIBUTOS DE UNA DOCENCIA DE CALIDAD EN LA EDUCACIÓN SUPERIOR: UNA REVISIÓN SISTEMÁTICA

\begin{tabular}{|c|c|c|c|c|}
\hline 童 & 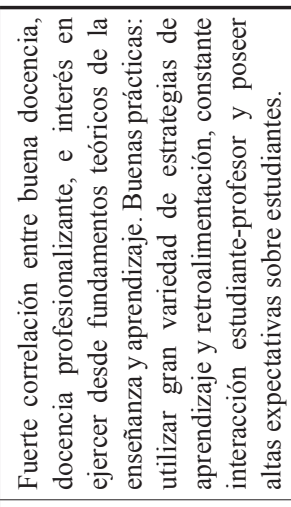 & 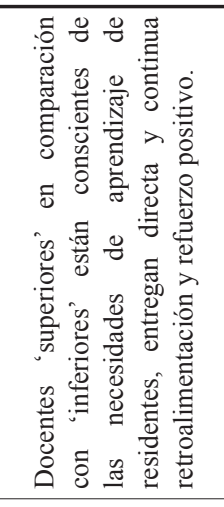 & 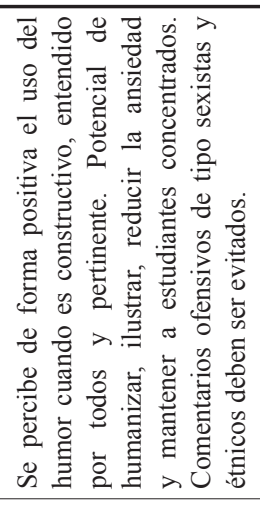 & 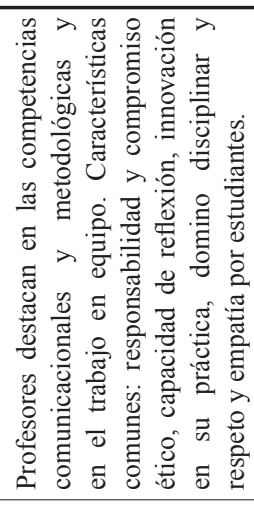 \\
\hline 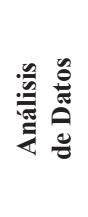 & 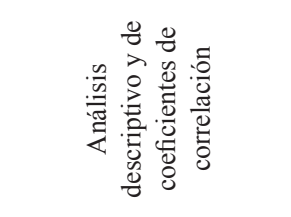 & 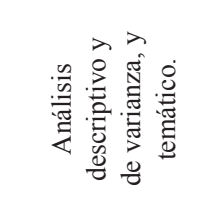 & 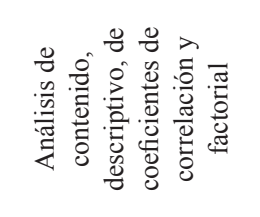 & 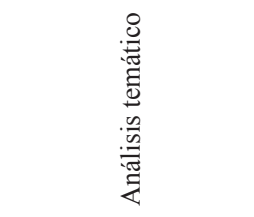 \\
\hline 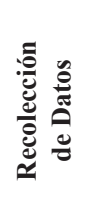 & 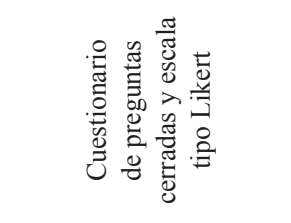 & 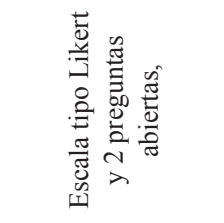 & 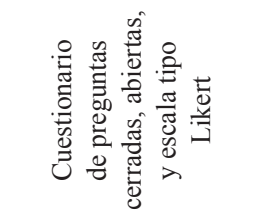 & 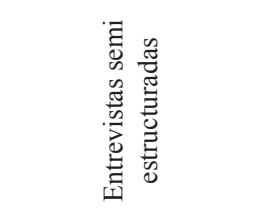 \\
\hline 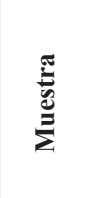 & 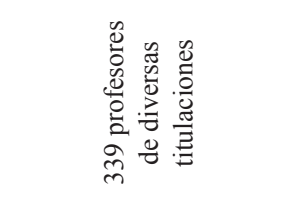 & 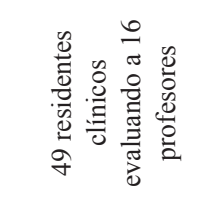 & 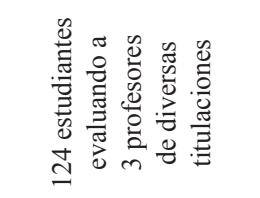 & 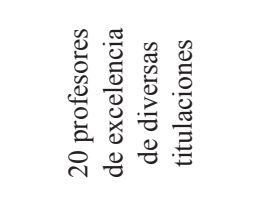 \\
\hline 产 & 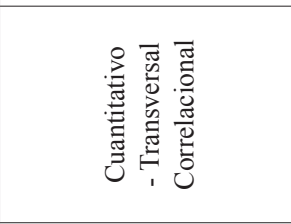 & 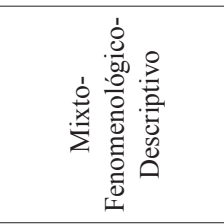 & 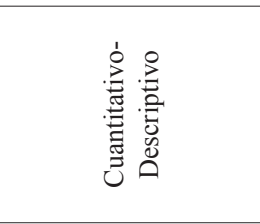 & 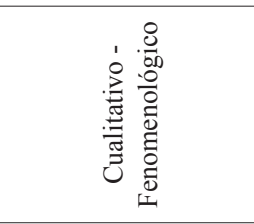 \\
\hline 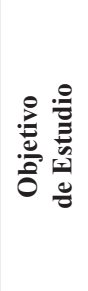 & 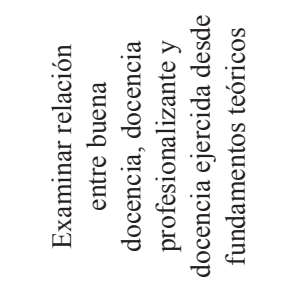 & 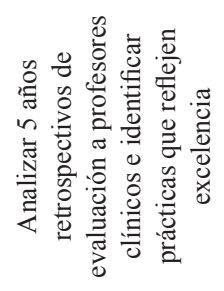 & 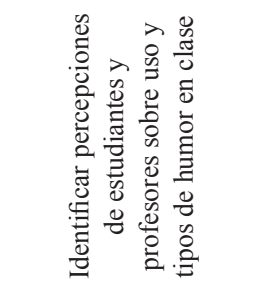 & 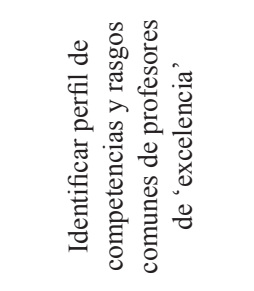 \\
\hline 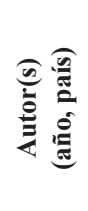 & 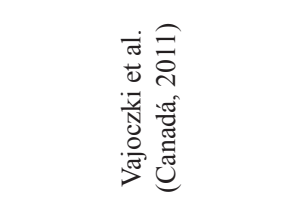 & 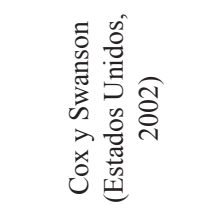 & 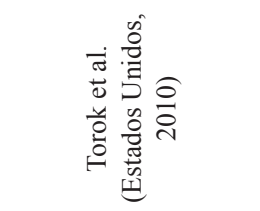 & 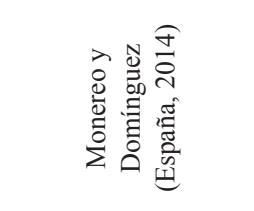 \\
\hline
\end{tabular}




\begin{tabular}{|c|c|c|c|c|c|}
\hline 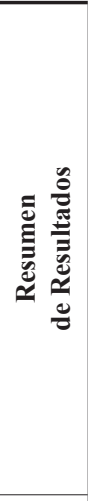 & 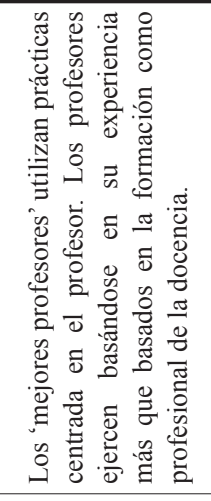 & 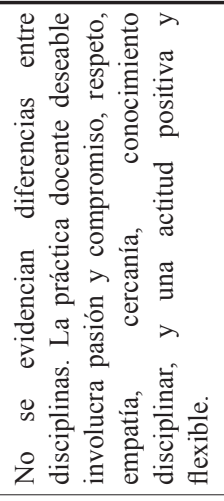 & 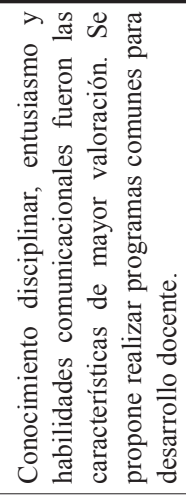 & 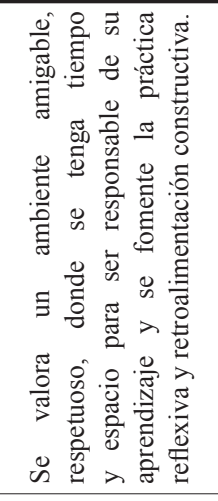 & 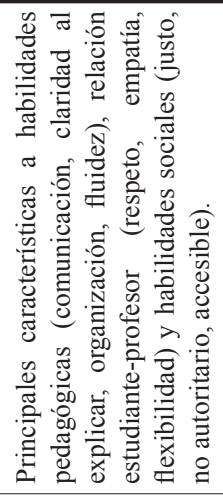 \\
\hline 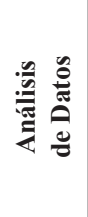 & 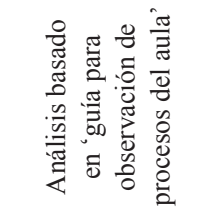 & 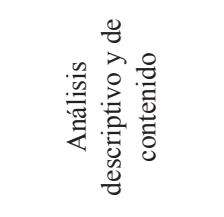 & 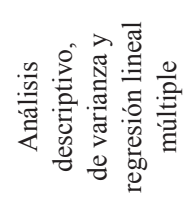 & 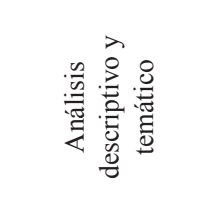 & 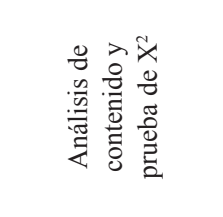 \\
\hline 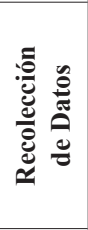 & 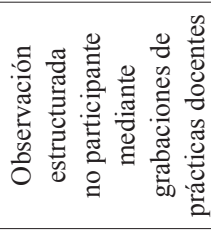 & 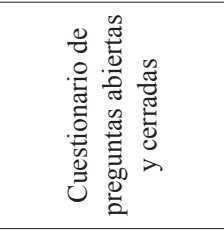 & 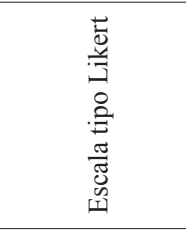 & 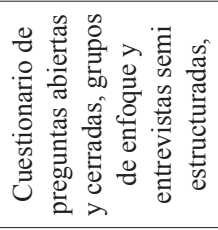 & 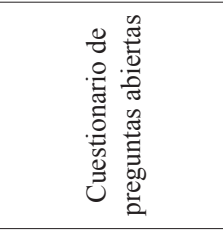 \\
\hline 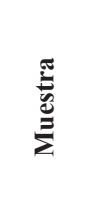 & 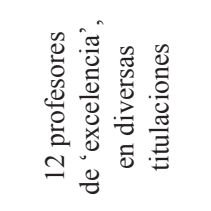 & 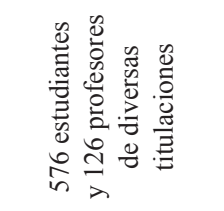 & 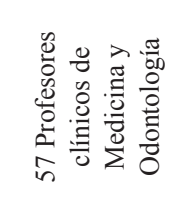 & 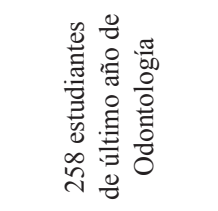 & 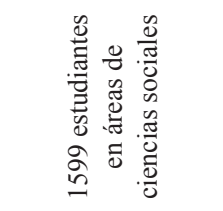 \\
\hline 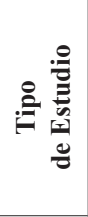 & 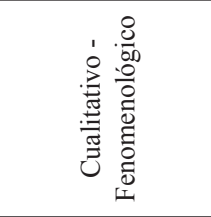 & 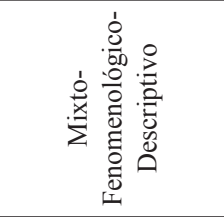 & 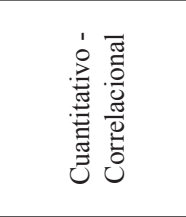 & 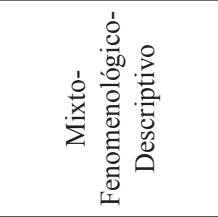 & 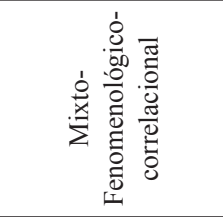 \\
\hline 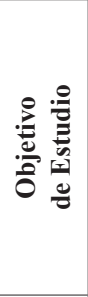 & 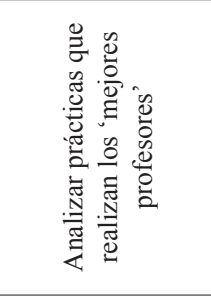 & 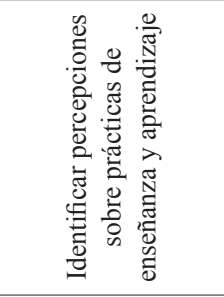 & 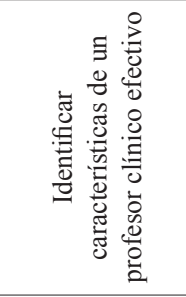 & 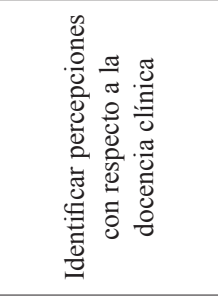 & 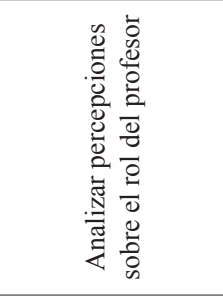 \\
\hline 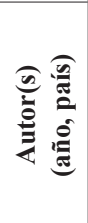 & 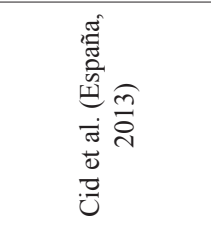 & 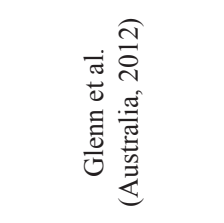 & 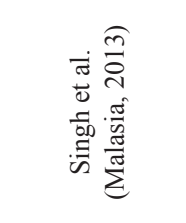 & 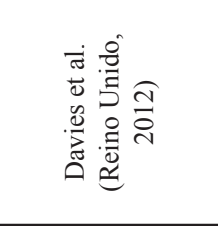 & 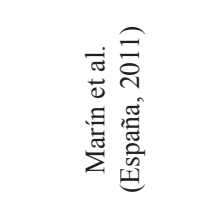 \\
\hline
\end{tabular}


Estudios Pedagógicos XLII, Nº 3: 483-506, 2016

ATRIBUTOS DE UNA DOCENCIA DE CALIDAD EN LA EDUCACIÓN SUPERIOR: UNA REVISIÓN SISTEMÁTICA

\begin{tabular}{|c|c|c|c|c|}
\hline 莺 & 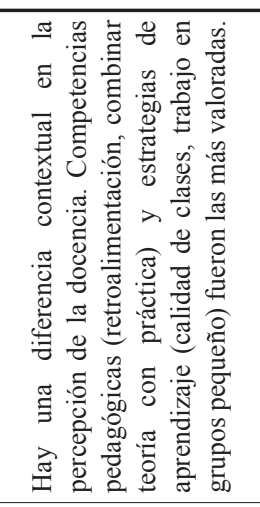 & 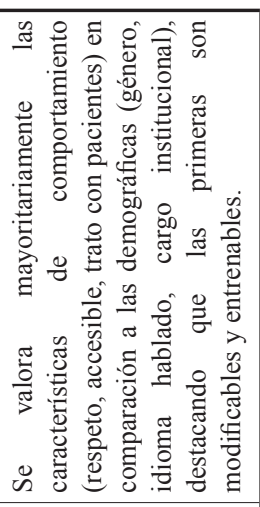 & 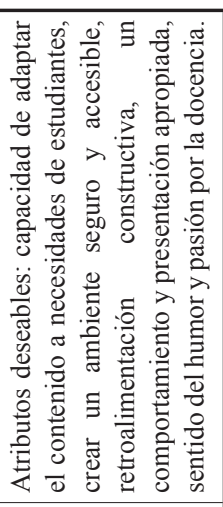 & 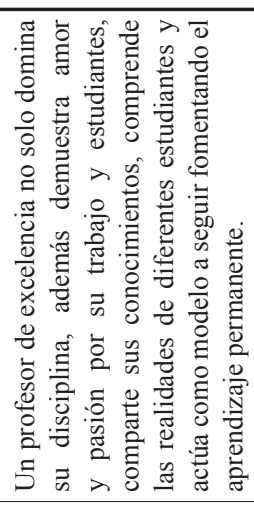 \\
\hline 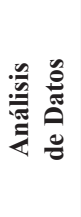 & 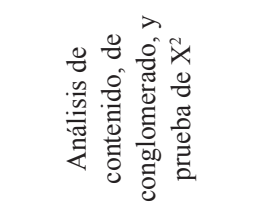 & 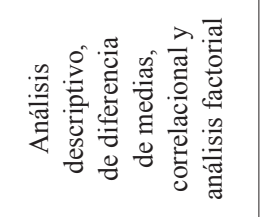 & 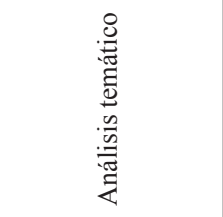 & 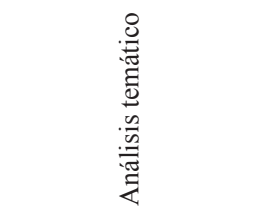 \\
\hline 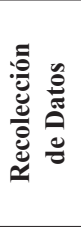 & 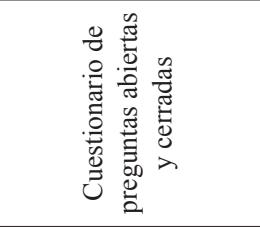 & 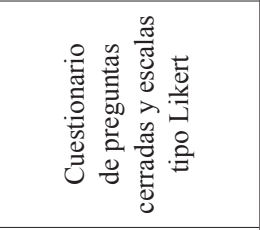 & 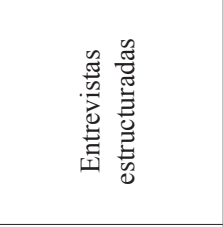 & 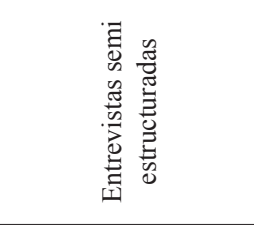 \\
\hline $\begin{array}{l}\frac{\mathbb{E}}{\mathrm{E}} \\
\stackrel{\Xi}{E}\end{array}$ & 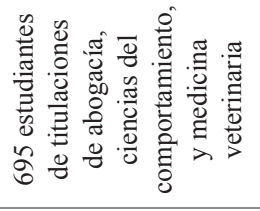 & 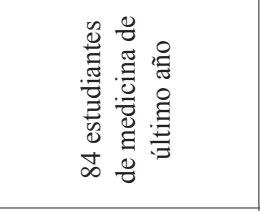 & 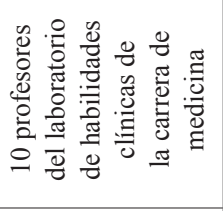 & 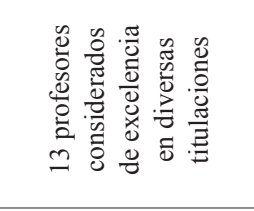 \\
\hline 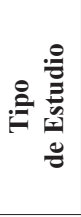 & 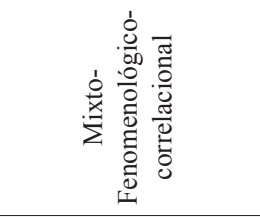 & 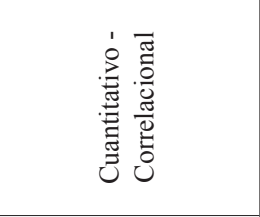 & 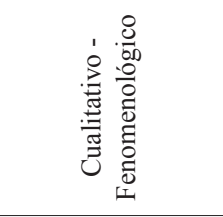 & 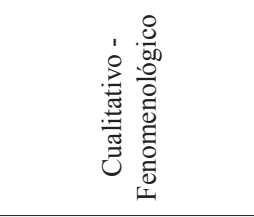 \\
\hline 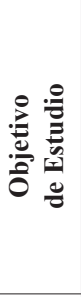 & 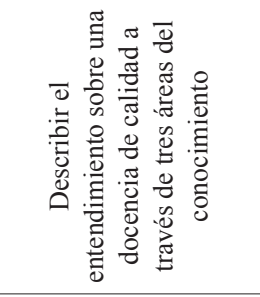 & 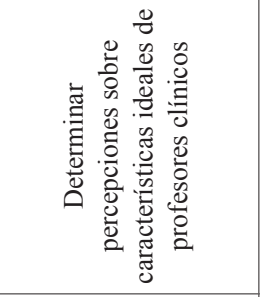 & 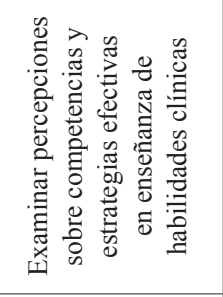 & 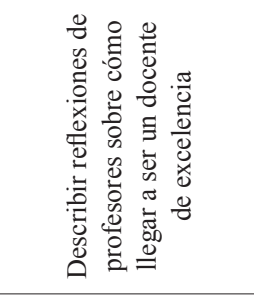 \\
\hline 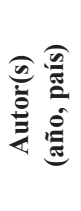 & 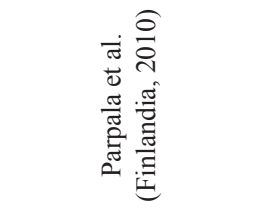 & 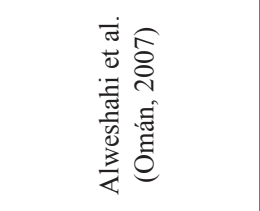 & 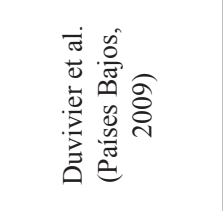 & 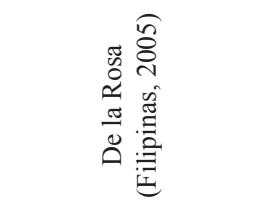 \\
\hline
\end{tabular}


Estudios Pedagógicos XLII, N³: 483-506, 2016 ATRIBUTOS DE UNA DOCENCIA DE CALIDAD EN LA EDUCACIÓN SUPERIOR: UNA REVISIÓN SISTEMÁTICA

\begin{tabular}{|c|c|c|c|}
\hline 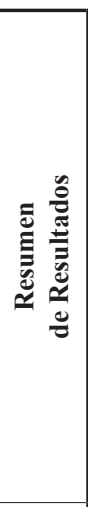 & 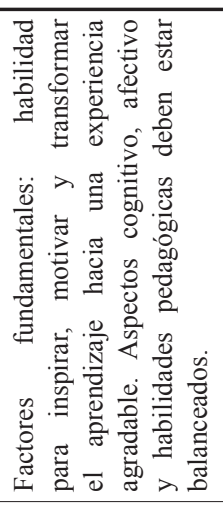 & 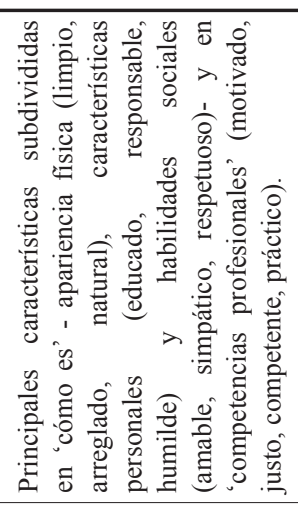 & 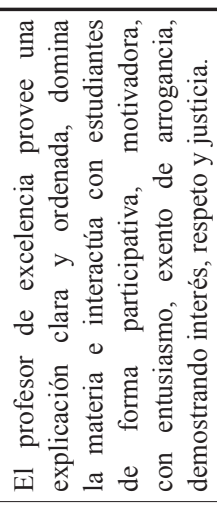 \\
\hline 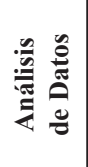 & 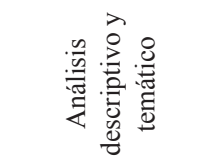 & 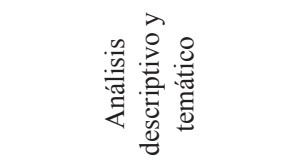 & 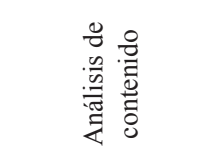 \\
\hline 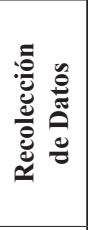 & 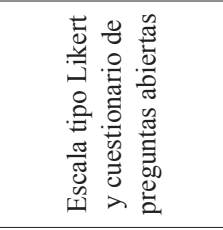 & 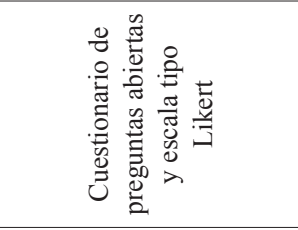 & 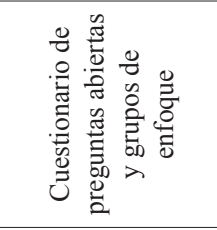 \\
\hline 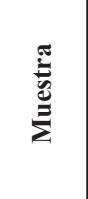 & 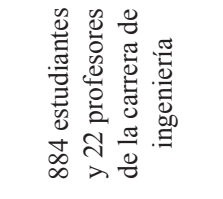 & 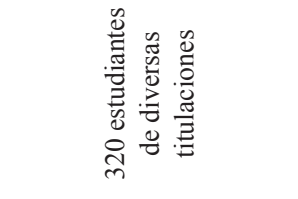 & 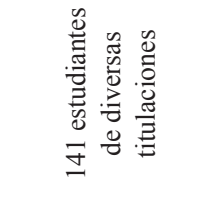 \\
\hline 总 & 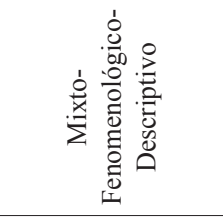 & 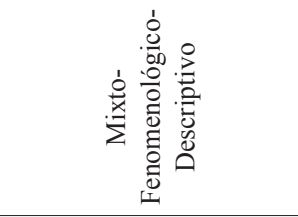 & 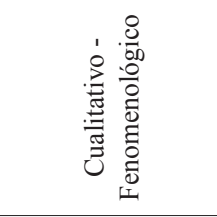 \\
\hline 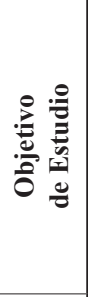 & 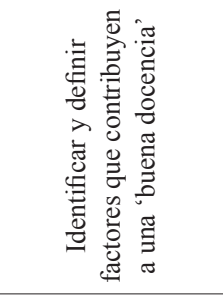 & 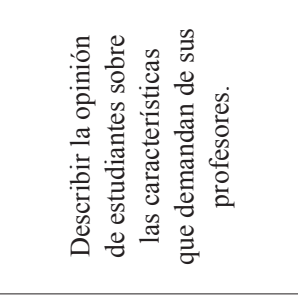 & 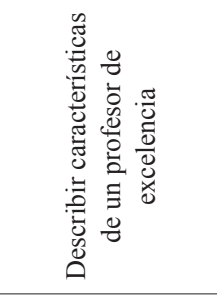 \\
\hline 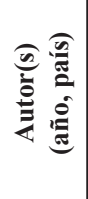 & 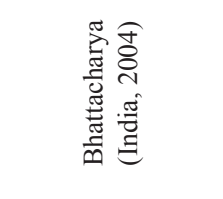 & 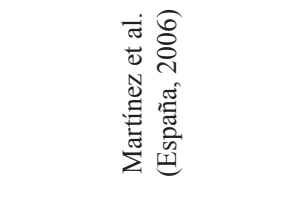 & 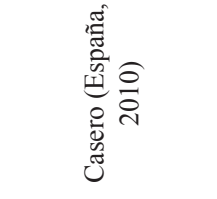 \\
\hline
\end{tabular}




\subsection{COMPETENCIAS GENÉRICAS}

Corresponden al conjunto de características, actitudes y conocimientos transversales que se requieren en cualquier área profesional, y que son transferibles a una gran variedad de ámbitos.

Las competencias genéricas del docente de excelencia se dividen en tres tipos (Tabla 4). Como parte de las características personales, estudiantes y profesores destacan la adecuada presentación del profesor, el mantener una buena apariencia (Duvivier et al., 2009; Martínez et al., 2006), el nivel de honestidad/sinceridad/transparencia del profesor (Bhattacharya, 2004) y, en menor medida, su capacidad intelectual (Marín et al., 2011). En el estudio realizado por Torok et al. (2010), 124 estudiantes de titulaciones de biología, psicología y teatro destacaron al profesor alegre, simpático y carismático, que hace uso de un humor constructivo. Se destaca en este estudio y en otros (Basow, et al., 2006; De la Rosa, 2005; Gargallo, 2008; Glenn et al., 2012; Martínez et al., 2006), que el humor debe ser entendido por todos y ser pertinente, teniendo así el potencial de humanizar, ilustrar, reducir la ansiedad y mantener la concentración de estudiantes. Historias, comentarios, humor profesional, e incluso el sarcasmo fueron formas muy bien evaluadas, mientras que comentarios de tipo ofensivos debiesen ser evitados.

Tabla 4. Atributos del docente de excelencia en la educación superior: competencias genéricas

\begin{tabular}{|c|c|c|c|}
\hline \multicolumn{4}{|c|}{ Competencias Genéricas } \\
\hline \multicolumn{2}{|c|}{$\begin{array}{l}\text { Características } \\
\text { Actitudinales }\end{array}$} & \multirow{2}{*}{\begin{tabular}{|l|}
\multicolumn{1}{|c}{$\begin{array}{c}\text { Características } \\
\text { Comunicativas }\end{array}$} \\
$\begin{array}{l}\text { Transmite información } \\
\text { de forma clara }\end{array}$ \\
\end{tabular}} & \multirow{2}{*}{\begin{tabular}{|c|}
\multicolumn{1}{|c}{$\begin{array}{c}\text { Características } \\
\text { Personales }\end{array}$} \\
$\begin{array}{l}\text { Posee sentido del } \\
\text { humor constructivo }\end{array}$
\end{tabular}} \\
\hline Creativo y persistente & Respetuoso & & \\
\hline Puntual & Motivador & $\begin{array}{l}\text { Escucha de forma } \\
\text { activa }\end{array}$ & $\begin{array}{l}\text { Mantiene una buena } \\
\text { apariencia }\end{array}$ \\
\hline Empático & $\begin{array}{l}\text { Conoce y se preocupa } \\
\text { por sus estudiantes }\end{array}$ & $\begin{array}{l}\text { Volumen y tono de voz } \\
\text { adecuado }\end{array}$ & $\begin{array}{l}\text { Honesto, íntegro y } \\
\text { transparente }\end{array}$ \\
\hline Positivo y optimista & Apoya a estudiantes & Asertivo & \multirow{2}{*}{ Carismático y sociable } \\
\hline $\begin{array}{l}\text { Humilde y acepta sus } \\
\text { limitaciones }\end{array}$ & $\begin{array}{l}\text { Genera confianza y } \\
\text { confía en estudiantes }\end{array}$ & $\begin{array}{l}\text { Interactúa con } \\
\text { estudiantes }\end{array}$ & \\
\hline $\begin{array}{l}\text { Valora el trabajo de } \\
\text { estudiantes }\end{array}$ & Paciente y atento & $\begin{array}{l}\text { Abierto y dispuesto a } \\
\text { responder preguntas }\end{array}$ & \multirow{2}{*}{$\begin{array}{l}\text { Emocional, social } \\
\text { y cognitivamente } \\
\text { inteligente }\end{array}$} \\
\hline $\begin{array}{l}\text { Compromiso y } \\
\text { seriedad con su trabajo }\end{array}$ & $\begin{array}{l}\text { Justo y consecuente en } \\
\text { su actuar }\end{array}$ & $\begin{array}{l}\text { Accesible y disponible } \\
\text { dentro y fuera de clase }\end{array}$ & \\
\hline $\begin{array}{l}\text { Responsable y } \\
\text { organizado }\end{array}$ & $\begin{array}{l}\text { Muestra amor, pasión e } \\
\text { interés en su trabajo }\end{array}$ & \multirow{2}{*}{$\begin{array}{l}\text { Receptivo a opiniones } \\
\text { de otros }\end{array}$} & \multirow{2}{*}{$\begin{array}{l}\text { Alegre, divertido y } \\
\text { simpático }\end{array}$} \\
\hline $\begin{array}{l}\text { Comprensivo y flexible } \\
\text { frente a necesidades de } \\
\text { estudiantes }\end{array}$ & $\begin{array}{l}\text { Actúa como modelo a } \\
\text { seguir }\end{array}$ & & \\
\hline
\end{tabular}


Por otra parte, las características actitudinales resultan altamente valoradas y parecen ser fundamentales a la hora de clasificar a un profesor como de 'excelencia'. Estas representan las características de determinados docentes que hacen que su comportamiento sea especialmente satisfactorio y valorado en el entorno educacional. El profesor de excelencia es descrito como responsable, organizado y puntual en los horarios de tutoría, demostrando compromiso y seriedad con su trabajo (Cabalín y Navarro, 2010; Gargallo 2008; Martínez et al., 2006). Es empático y respetuoso en su actuar, mostrando capacidad para ponerse en el lugar de sus estudiantes y valorar sus trabajos (Cabalín y Navarro, 2010; Gargallo, 2008; Glenn et al., 2012; Jahangiri et al., 2013; Monereo y Domínguez, 2014). Es más, las grabaciones del comportamiento de profesores de excelencia realizadas por Hativa et al. (2001) reflejan un clima positivo caracterizado por llevar una actitud optimista y de apoyo a los estudiantes (Basow et al., 2006; Cox y Swanson, 2002; Jahangiri et al., 2013; Martínez et al., 2006), resultando en la generación de entusiasmo y motivación para aprender (Bhattacharya, 2004; Singh et al., 2013). Estudiantes de titulaciones tan diversas como las provenientes de áreas de ciencias sociales y ciencias médicas destacan que sus profesores de excelencia generan confianza y confían en sus estudiantes, y son flexibles y comprensivos frente a las diversas situaciones que ellos enfrentan (Alweshahi et al., 2007; Bartram y Bailey 2009; Basow et al., 2006; Martínez et al., 2006; Monereo y Domínguez 2014).

Otro factor importante es la humildad del profesor, desde una actitud exenta de arrogancia (Basow et al., 2006; García et al., 2006; Glenn et al., 2012; Martínez, 2010). Así también lo describe Marín et al. (2011) en un estudio donde 1599 estudiantes de pregrado de áreas de ciencias sociales manifestaron la importancia del profesor universitario en ser justo, no autoritario ni arrogante, y sin mostrarse esquivo cuando los estudiantes necesitan asistencia, como por ejemplo en materias que superen su conocimiento. Así, el mensaje del profesor es recibido con mayor credibilidad y no genera dudas, confusiones ni resistencia (Monereo y Domínguez, 2014). También existe evidencia de lo crucial que es el componente afectivo del profesor (Glenn et al., 2012; Monereo y Domínguez, 2014). Así lo refleja De la Rosa (2005) a través de las reflexiones sobre su práctica de profesores considerados como de excelencia y cómo llegaron a ser considerados como tales, destacan que no solo se debe dominar la disciplina, sino que además demostrar amor y pasión por su trabajo y ser conscientes sobre su responsabilidad como modelo a seguir (Davies et al., 2012; Duvivier et al., 2009).

Finalmente, las características comunicativas están destinadas a facilitar la comprensión mediante estrategias discursivas, ajustándose al auditorio y favoreciendo que los estudiantes puedan expresarse libremente, siendo escuchadas y respetadas sus opiniones (Monereo y Domínguez, 2014). Bartram y Bailey (2009) reportan que 268 estudiantes británicos y extranjeros, independiente de su nacionalidad, catalogaron como 'docencia efectiva' la ejercida por un profesor que explica y transmite la información de forma clara. Similares estudios destacan las cualidades de un profesor que se expresa con un volumen y tono de voz adecuado, con el objetivo de comunicar bien para que le comprendan mejor (De la Rosa, 2005; Gargallo, 2008; Hativa et al., 2001; Monereo y Domínguez, 2014; Marín et al., 2011).

Sin embargo, no solo es cuestión de 'hablar' en forma efectiva, sino también el escuchar a los estudiantes de forma activa y ser receptivo a sus opiniones (Basow et al., 2006). Al analizar los métodos de enseñanza de 42 profesores, Gargallo et al. (2010) señalan que 
los estudiantes cuyos profesores trabajan con metodologías centradas en el aprendizaje, exhibían mejores actitudes. Dentro de ellas el mostrarse 'abierto', dispuesto a responder preguntas y asertivos en sus comentarios. Esto es también evidente en la docencia clínica, donde Jahangiri et al. (2013) identifican entre las características de un docente clínico efectivo el ser accesible, dentro y fuera de clases, y el hecho de preocuparse de interactuar en forma constante con sus estudiantes.

\subsection{COMPETENCIAS PEDAGÓGICAS}

Las competencias pedagógicas (Tabla 5) resultaron ser las con mayor peso relativo, y se refieren al conjunto de habilidades, destrezas y aptitudes que se desarrollan y despliegan exclusivamente en el contexto pedagógico del docente e involucran principalmente a los métodos utilizados para facilitar el aprendizaje; y a las prácticas de planificación-gestión de la docencia (Monereo y Domínguez, 2014). Esto es independiente del área disciplinar del profesor, y se refiere a la formación de carácter pedagógica y aplicación de esta a su quehacer diario.

En primer lugar, las estrategias de enseñanza-aprendizaje se refieren a las prácticas docentes como herramientas, tanto teóricas como metodológicas, sobre aspectos necesarios para la inclusión de todos los estudiantes en las aulas universitarias. Para lograr esta inclusión, los estudiantes consideran fundamental que el profesor potencie el aprendizaje significativo y centrado en el estudiante, a través de diversas estrategias de aprendizaje que sean activas, y se adecúen a sus intereses y características (Cox y Swanson, 2002; Duvivier et al., 2009; Friz et al., 2011; Gargallo, 2008). Así lo reporta Vajoczki et al. (2011), en un estudio donde 339 profesores considerados como 'buenos' y 'ejerciendo con fuerte base pedagógica', manifestaron el uso de diversas estrategias de aprendizaje, tales como tutoriales con grupos pequeños, discusión guiada, aprendizaje entre pares y aprendizaje basado en problemas. Resulta destacable que la tradicional clase magistral solo fue elegida por estudiantes y profesores en un mínimo número de casos, y solo cuando era estrictamente necesaria (Gargallo, 2008; Glenn et al., 2012). Es más, en Australia, 126 profesores de diversas titulaciones consideraron que la clase magistral no provee una buena experiencia de aprendizaje, al desalentar la participación del estudiante y no proveer una forma de aprendizaje activo (Glenn et al., 2012). Ellos mismos manifiestan que "los estudiantes aprenden a pesar de tener clases magistrales y no debido a ellas" (Glenn et al., 2012, p. 206).

La profesores que trabajan con metodologías centradas en facilitar el aprendizaje desde el modelo constructivista centrado en el estudiante y con altas expectativas de lo que sus estudiantes pueden lograr, muestran evidencias de que sus estudiantes desarrollan estrategias de aprendizaje de mayor calidad y profundidad, teniendo un rendimiento académico superior comparado a estudiantes cuyos profesores trabajan con metodologías centradas en la enseñanza, de tipo tradicional (Gargallo et al., 2010). Es más, Friz et al. (2011) describen que 81 estudiantes chilenos de pedagogía en matemática consideran como fundamentales dentro de sus competencias profesionales el crear un clima participativo en el cual se fomente equitativamente el trabajo y discusión grupal y el trabajo individual; visión compartida por estudiantes estadounidenses (Basow et al., 2006), británicos (Bartram y Bailey, 2009) y españoles (Casero, 2010). 
Tabla 5. Atributos del docente de excelencia en la educación superior: competencias pedagógicas

\begin{tabular}{|l|l|l|l|}
\hline \multicolumn{3}{|c|}{ Competencias Pedagógicas } \\
\hline \multicolumn{2}{|c|}{ Estrategias de Ensenanza-Aprendizaje } & \multicolumn{2}{|c|}{ Planificación-Gestión } \\
\hline $\begin{array}{l}\text { Provee experiencia } \\
\text { de aprendizaje } \\
\text { vicaria }\end{array}$ & $\begin{array}{l}\text { Facilita } \\
\text { pensamiento crítico } \\
\text { y auto evaluación }\end{array}$ & $\begin{array}{l}\text { Explica los resultados de } \\
\text { aprendizaje }\end{array}$ & $\begin{array}{l}\text { Propone problemas reales } \\
\text { provenientes del contexto } \\
\text { profesional }\end{array}$ \\
\hline $\begin{array}{l}\text { Altas expectativas } \\
\text { de sus estudiantes }\end{array}$ & $\begin{array}{l}\text { Actúa como guía y } \\
\text { mentor }\end{array}$ & $\begin{array}{l}\text { Prepara apuntes de } \\
\text { calidad ajustados a } \\
\text { resultados de aprendizaje }\end{array}$ & $\begin{array}{l}\text { Evaluación coherente con } \\
\text { actividades de aprendizaje }\end{array}$ \\
\hline $\begin{array}{l}\text { Responde consultas } \\
\text { a tiempo }\end{array}$ & $\begin{array}{l}\text { Crea clima positivo } \\
\text { y participativo }\end{array}$ & $\begin{array}{l}\text { Considera la carga de } \\
\text { trabajo del estudiante }\end{array}$ & $\begin{array}{l}\text { Implementa actividades de } \\
\text { evaluación formativas y } \\
\text { sumativas }\end{array}$ \\
\hline $\begin{array}{l}\text { Considera } \\
\text { conocimientos } \\
\text { previos de } \\
\text { estudiantes }\end{array}$ & $\begin{array}{l}\text { Da refuerzo y } \\
\text { retroalimentación } \\
\text { positiva y } \\
\text { constructiva }\end{array}$ & $\begin{array}{l}\text { Reflexiona sobre su } \\
\text { práctica docente }\end{array}$ & $\begin{array}{l}\text { Prepara y organiza las } \\
\text { actividades de aprendizaje }\end{array}$ \\
\hline $\begin{array}{l}\text { Fomenta la } \\
\text { discusión entre } \\
\text { estudiantes }\end{array}$ & $\begin{array}{l}\text { Promueve la } \\
\text { autonomía del } \\
\text { estudiante }\end{array}$ & $\begin{array}{l}\text { Conoce implicaciones } \\
\text { educativas de TiCs }\end{array}$ & Innova en su metodología \\
\hline $\begin{array}{l}\text { Versátil, presenta actividades de forma } \\
\text { interesante }\end{array}$ & $\begin{array}{l}\text { Proporciona referencias } \\
\text { bibliográficas precisas, y } \\
\text { diversas fuentes de } \\
\text { aprendizaje }\end{array}$ & $\begin{array}{l}\text { Explica criterios y } \\
\text { procedimientos de } \\
\text { evaluación }\end{array}$ \\
\hline $\begin{array}{l}\text { Panifica actividades de } \\
\text { evaluación flexibles, con } \\
\text { opciones y variedades } \\
\text { simplificados } \\
\text { activas }\end{array}$ & $\begin{array}{l}\text { Existe coherencia entre } \\
\text { resultados y actividades } \\
\text { de aprendizaje, y } \\
\text { actividades de evaluación }\end{array}$ & $\begin{array}{l}\text { Ponoce y comprende los } \\
\text { entenidos y organización } \\
\text { evaluaciones currículum }\end{array}$ \\
\hline
\end{tabular}

Otro factor que diferencia a un profesor de excelencia es el promover el refuerzo positivo y la retroalimentación constructiva y a tiempo, tanto de forma grupal como individualizada, y tanto para las actividades de aprendizaje como para las de evaluación (Alweshahi et al., 2007; Basow et al., 2006; Duvivier et al., 2009; Hativa et al., 2001; Parpala et al., 2011; Vajoczki et al., 2011). En el estudio de Cox y Swanson (2002), se analizaron 5 años retrospectivos de evaluaciones de 46 residentes de medicina a 16 profesores clínicos; como resultado, los profesores 'superiores', en comparación con los 'inferiores', se caracterizaron por proveer tanto en clínica como en pabellón un constante refuerzo positivo y una retroalimentación de carácter directa y continua sobre el progreso de los residentes. 
El proveer al estudiante con herramientas para asegurar la autonomía de su aprendizaje como finalidad última, se describe a través de la facilitación de habilidades reflexivas, críticas y de auto evaluación, y también a través de la capacidad de pensamiento creativo del docente (Alweshahi et al., 2007; Bartram y Bailey, 2009; Bhattacharya, 2004; Davidovitch y Milgram, 2006; Hativa et al., 2001; Monereo y Domínguez, 2014; Parpala et al., 2011).

A raíz de lo anterior se desprende la relevancia del actuar del profesor como constante guía y mentor (Bartram y Bailey, 2009), tomándose este el tiempo necesario para entregar explicaciones y ejemplos de forma ordenada y simplificada (Bartram y Bailey, 2009; Cid et al., 2013; Marín et al., 2011). Es más, en los estudios de Duvivier et al. (2009) y De la Rosa (2005) se destaca la experiencia vicaria que proveen los profesores, mostrando y demostrando habilidades desde la práctica y no solamente a través del discurso.

Por otra parte, y dentro de las mismas competencias pedagógicas, se encuentra la planificación y gestión de la docencia. Esto se refiere a temas como la selección de contenidos de acuerdo con su relevancia en la futura profesión, como también el diseñar y desarrollar procesos de enseñanza y evaluación pertinentes con la planificación docente, y con detectar puntos débiles en la propia docencia con el fin de mejorarlos (Monereo y Domínguez, 2014).

En primer lugar, se destaca la dedicación y el tiempo destinado a la preparación y organización de las actividades de aprendizaje (Cid et al., 2013; Friz et al., 2011), y también el ocupar recursos de apoyo como las tecnologías de la información y la comunicación (Friz et al., 2011; Gargallo, 2008; De la Rosa, 2005). Dentro de las cualidades pedagógicas de sus profesores, 37 de 50 estudiantes españoles en el estudio de Gargallo (2008) mencionaron como la segunda más valorada, precisamente, a la dedicación del profesor a preparar las clases. Además, Bhattacharya (2004) destaca otro aspecto relevante: el explicar y discutir con los estudiantes sobre los resultados de aprendizaje esperados. También resulta clave proporcionar apuntes, referencias bibliográficas precisas, y recursos de aprendizaje adicionales (Alweshahi et al., 2007; Gargallo 2008).

Finalmente, otro aspecto también relacionado con la planificación de las actividades de aprendizaje es que el profesor proponga problemas derivados directamente del contexto profesional, estableciendo así una base lógica entre las futuras acciones profesionales y los temas de la asignatura (Gargallo, 2008). Monereo y Domínguez (2014) destacan como uno de los rasgos comunes entre los 20 profesores participantes de su estudio y considerados como de excelencia, el actuar muy orientados hacia la enseñanza funcional, valorando positivamente las actividades prácticas, de terreno y de aplicación a problemas reales, extraídos del ejercicio profesional.

En segundo lugar, se destaca la planificación de actividades de evaluación, siendo estas flexibles y ofreciendo diversas opciones y variedades. En efecto, estudiantes españoles valoraron la ausencia del tradicional 'examen final', y que en su lugar haya una evaluación continua, con actividades de evaluación a lo largo de todo el semestre basadas en sus trabajos, su progreso y esfuerzo.

Resulta además importante la coherencia que tengan estas actividades de evaluación con lo trabajado en las mismas actividades de aprendizaje, de ahí también la importancia de explicar y dar a conocer los diferentes criterios y procedimientos de evaluación (Casero 2010; Parpala et al., 2011). En suma, al implementar estas medidas se logra facilitar el aprendizaje estableciendo una alineación entre los resultados de aprendizaje, actividades de aprendizaje y actividades de evaluación (Glenn et al., 2012). 
Finalmente, el profesor de excelencia también gestiona sobre su propia docencia, el reflexionar sobre sus aciertos y errores, evaluar su propia enseñanza y el aprendizaje de sus estudiantes y la preocupación por la innovación metodológica han sido citados como competencias esenciales para promover la formación del profesorado universitario (De la Rosa 2005; Monereo y Domínguez 2014). Igualmente, resulta clave dentro de esta 'auto gestión' el comprender los contenidos y organización del currículum de la titulación, para así también comprender y considerar la carga de trabajo de los estudiantes (Duvivier et al., 2009).

\subsection{COMPETENCIAS DISCIPLINARES}

El último tema emergido, con el menor peso relativo y de mayor importancia para los profesores que para los estudiantes, se relaciona con las competencias disciplinares (Tabla 6). Estas se refieren a los conocimientos, habilidades y actitudes del profesor, considerados como los mínimos necesarios dentro de cada campo disciplinar que se está enseñando (Friz et al., 2011).

Tabla 6. Atributos del docente de excelencia en la educación superior: competencias disciplinares

\begin{tabular}{|l|}
\hline \multicolumn{1}{|c|}{ Competencias disciplinares } \\
\hline Experto en su área del conocimiento \\
\hline Actualizado y preocupado por su formación continua \\
\hline Vasta experiencia profesional \\
\hline Dominio cognoscitivo y práctico del contenido que enseña \\
\hline Desarrolla el componente investigador para alimentar su saber \\
\hline
\end{tabular}

El primer aspecto destacado es el dominio cognoscitivo y práctico del contenido que se enseña (Basow et al., 2006; Bhattacharya, 2004; Cox y Swanson, 2002; De la Rosa, 2005; Friz et al., 2011; Gargallo, 2008; Marín et al., 2011; Jahangiri et al., 2013). Por ejemplo, en el estudio de Cabalín y Navarro (2010), 104 docentes y 293 estudiantes de diversas titulaciones del área de la salud, a través de un análisis de redes semánticas y con la palabra estímulo 'buen profesor', el concepto de 'conocimiento' se ubicó dentro del segundo y tercer núcleo de palabras relevantes, respectivamente. Por lo tanto el ser un 'experto en su área de conocimiento' y específicamente en 'la materia que enseña' es valorado tanto por estudiantes como por profesores (Friz et al., 2011; Glenn et al., 2012; Marín et al., 2011; Singh et al., 2013). Sin embargo, no solo es necesario tener un vasto conocimiento teórico, sino que también resulta relevante poseer una vasta experiencia profesional, ya sea a través de estudios formales o a través de la experiencia profesional (Marín et al., 2011).

El segundo aspecto que resalta es el estar actualizado, preocupado por su formación continua y el desarrollar el componente investigador (Cabalín y Navarro, 2010; Martínez et al., 2006; Monereo y Domínguez, 2014). Docentes resaltan el hecho de conocer a cabalidad 
su área de estudio y estar preparado para mantener su nivel de conocimiento en el tiempo a través de la formación continua e investigación (Glenn et al., 2012).

\section{DISCUSIÓN Y CONCLUSIONES}

El propósito del presente estudio ha sido realizar una revisión sistemática descriptiva de la literatura sobre los atributos de una docencia de calidad, destacando los aspectos provenientes de las opiniones y reflexiones de estudiantes y profesores en diversos contextos, y considerando la evidencia generada con base en las prácticas de profesores considerados como de excelencia.

Podemos afirmar que los estudios sobre calidad de la docencia universitaria cobran relevancia académica para aumentar la comprensión, proyección y mejoramiento continuo del sistema universitario, y que este tipo de estudios requieren de los contextos particulares de cada sistema educativo (Bain, 2004; Hannah et al., 2011). En gran parte de los estudios revisados, las investigaciones son acotadas a una misma institución y disciplina; se han centrado más en la percepción de estudiantes y docentes, que en las prácticas de docentes de excelencia.

Por un lado, gran parte de los estudios se basa en el acceso a través del discurso y mediante respuesta a reactivos, con los estudiantes y profesores narrando y analizando sus prácticas. Si bien esto permite acceder a percepciones y prácticas declaradas, tal 'declaración' no siempre coincide con el actuar o con lo que realmente se quiere decir o se quiere ocultar (Bain, 2004). Por lo tanto, y sin negar el valor de las prácticas declaras, estas no siempre reflejan la realidad. Solo algunos estudios triangularon sus métodos en busca de otros enfoques, como la observación y análisis de documentos, centrados en la identificación y análisis de las buenas prácticas (Cid et al., 2013; Hativa et al., 2001). Es recomendable que futuras investigaciones centradas en atributos de una buena docencia, incorporen tanto prácticas 'declaradas' como prácticas 'observadas'.

El análisis realizado ha identificado tres grandes grupos de competencias que definen los atributos de una docencia de calidad. Sin embargo, el categorizarlas en competencias genéricas, pedagógicas y disciplinares (Figura 2), no resultó ser tarea fácil. Si bien cada competencia tiene particulares características y fines, resulta evidente que muchos de los indicadores categorizados en un tema podrían formar parte de otro. En parte, esto pudiese relacionarse a que una docencia de calidad es un fenómeno multifacético (Hativa et al., 2001), y la categorización y división de los atributos continúa siendo, en parte, ambigua. La fuerte sobreposición entre los diferentes indicadores es muestra de lo interrelacionadas que son las diferentes competencias definidas. A nuestro parecer, estas forman un todo y son divididas por razones didácticas, es decir, son complementarias para lograr una docencia de excelencia.

Por otra parte, la categorización propuesta busca facilitar la comprensión de los atributos integrales del profesor de excelencia. Las tres diferentes áreas han sido valoradas por estudiantes y profesores en diversas latitudes y en diversas áreas de la educación superior, justificando de esta forma su categorización. Además, estos resultados tienen el potencial de ser utilizados para el desarrollo de instrumentos, tanto para la evaluación de la efectividad docente, para el criterio de reclutamiento y promoción de profesores, como para la planificación de estrategias e intervenciones de desarrollo docente. Es más, 
se concluye que no hay claras diferencias entre disciplinas y que este grupo de atributos sería transversal, independiente del área de estudio (Glenn et al., 2012; Singh et al., 2013).

$\mathrm{Si}$ bien es cierto que algunos atributos son de mayor relevancia dependiendo del contexto en el que se desenvuelva el docente (Parpala et al., 2011), el conjunto de atributos es transversal y pertinente a ser presentado en programas comunes de desarrollo docente independiente del área de estudio, tomando en cuenta la propia reflexión del docente sobre cuáles atributos debe potenciar.

En cuanto a la valoración que otorgaron estudiantes y profesores a los tres grupos de competencias identificadas, las competencias pedagógicas fueron las más valoradas, seguidas por las competencias genéricas y por las competencias disciplinares. Por una parte, los estudiantes otorgan mayor valor a las competencias pedagógicas y a las competencias genéricas (Alweshahi et al., 2007; Bartram y Bailey, 2009; Davies et al., 2012; Friz et al., 2011; Glenn et al., 2012; Jahangiri et al., 2013; Marín et al., 2011; Monereo y Domínguez, 2014; Parpala et al., 2011), y los profesores a las competencias disciplinares (Cabalín y Navarro, 2010; Casero, 2010; Singh et al., 2013). Los estudiantes valoran más el cómo el profesor transmite los conocimientos y cómo les facilita el aprendizaje, sin embargo, esa facilitación requiere además de competencias disciplinares. Por lo tanto, se hace fundamental que el buen profesor maneje a cabalidad el contenido de los resultados de aprendizaje que espera lograr en sus estudiantes. Se puede argumentar que una de las razones por las cuales las competencias disciplinares no destacan en la opinión de los estudiantes, es porque ellos dan por asumido y parten de la base de que los profesores 'saben', en cambio los profesores están más conscientes de sus propias limitaciones en el 'saber'. Otro factor a considerar en esta subvaloración de las competencias disciplinares por parte del estudiantado es el cambio en el paradigma del profesor como contenedor de todo el conocimiento, incorporando una visión del docente como facilitador, que no lo sabe 'todo' pero sí es capaz de crear las condiciones para que sus estudiantes exploren, aprendan y potencien sus intereses (Bartram y Bailey, 2009).

Estudiantes y profesores resaltan la necesidad fundamental de prepararse para ser profesor universitario. Resulta insuficiente ser un profesional competente y resulta indispensable incorporar competencias pedagógicas para lograr el máximo desarrollo en los estudiantes (Alweshahi et al., 2007; Singh et al., 2013). Así también lo son las competencias genéricas; los aspectos actitudinales y comunicativos son determinantes a la hora de relacionarse con los estudiantes. No solo resulta clave la inteligencia cognitiva, sino también la inteligencia emocional y social, determinantes a la hora de decidir si un profesional está preparado para desempeñar la función docente.

A pesar de la rigurosidad empleada en el análisis de la literatura seleccionada y de los relevantes resultados obtenidos, es necesario destacar las limitaciones propias de la presente revisión. En primer lugar, la revisión está limitada a las fuentes revisadas e importantes estudios pueden haber sido excluidos. En segundo lugar, otra fuente de sesgo proviene del hecho que solamente se tuvieran en cuenta estudios redactados en los idiomas español e inglés, descartándose la literatura publicada en otros idiomas. En tercer lugar, la diversidad de diseños, muestras, análisis e instrumentos de medida empleados en los artículos analizados limitaron la revisión a ser principalmente descriptiva, descartando el meta análisis de datos. Finalmente, la selección, análisis y codificación de datos estuvo sujeta al criterio de los autores, sin embargo, se emplearon métodos para aumentar la credibilidad y confiabilidad de los resultados, tales como el uso de programas computacionales 
especializados, revisión independiente y chequeos cruzados y mediaciones de terceros en caso de desacuerdo (Hernández et al., 2010).

En suma, los resultados de este estudio son de especial relevancia para profesores, directivos y formadores del profesorado universitario. Las características identificadas, en general, son factibles de ser modificadas, aprendidas y entrenadas. Futuras investigaciones debieran continuar el estudio de los componentes de calidad en la educación superior, especialmente en lo que concierne a las características del docente y su impacto en el aprendizaje de los estudiantes. El identificar los atributos de una docencia de calidad y aplicarlas a cualquier intervención que aspire conseguir un aprendizaje significativo y de calidad deberá, por tanto, tener presente el papel crucial que juegan las variables de tipo genéricas, pedagógicas y disciplinares en la interacción profesor-estudiante.

\section{REFERENCIAS BIBLIOGRÁFICAS}

AACSB. (2015). Eligibility Procedures and Accreditation Standards for Business Accreditation. AACSB International - The Association to Advance Collegiate Schools of Business.

AMBA. (2015). Criteria for Accreditation of MBA programmes.

Alweshahi, Y., Harley, D., \& Cook, D. A. (2007). Students' perception of the characteristics of effective bedside teachers. Medical Teacher, 29(2/3), 204-209.

Bain, K. (2004). What the best college teachers do? Cambridge: Harvard University Press.

Bartram, B., \& Bailey, C. (2009). Different students, same difference?: A comparison of UK and international students' understandings of 'effective teaching'. Active Learning in Higher Education, 10(2), 172-184.

Basow, S. A., Phelan, J. E., \& Capotosto, L. (2006). Gender patterns in college students' choices of their best and worst professors. Psychology of Women Quarterly, 30(1), 25-35.

Bhattacharya, B. (2004). What is 'good teaching' in engineering education in India? A case study. Innovations in Education and Teaching International, 41(3), 329-341.

Cabalín, D., \& Navarro, N. (2010). Conceptualización de los estudiantes sobre el buen profesor universitario en las carreras de la salud de la Universidad de La Frontera - Chile. Int. J. Morphol, $26,887-892$.

Casero, A. (2010). ¿Cómo es el buen profesor universitario según el alumnado? Revista Española de Pedagogía, 68(246), 223-242.

Cid, A., Pérez, A., \& Zabalza, M. A. (2013). Las prácticas de enseñanza realizadas/observadas de los "mejores profesores" de la Universidad de Vigo. Educación XX1, 16(2), 265-296.

Cox, S. S., \& Swanson, M. S. (2002). Identification of teaching excellence in operating room and clinic settings. American Journal of Surgery, 183(3), 251-255.

Davidovitch, N., \& Milgram, R. M. (2006). Creative thinking as a predictor of teacher effectiveness in higher education. Creativity Research Journal, 18(3), 385-390.

Davies, B., Leung, A., \& Dunne, S. (2012). So how do you see our teaching? Some observations received from past and present students at the Maurice Wohl Dental Centre. European Journal of Dental Education, 16(3), 138-143.

De la Rosa, P. S. M. (2005). Toward a more reflective teaching practice: Revisiting excellence in teaching. Asia Pacific Education Review, 6(2), 170-176.

Devlin, M., \& Samarawickrema, G. (2010). The criteria of effective teaching in a changing higher education context. Higher Education Research \& Development, 29(2), 111-124.

Duvivier, R. J., Van Dalen, J., Van der Vleuten, C. P. M., \& Scherpbier, A. J. J. A. (2009). Teacher perceptions of desired qualities, competencies and strategies for clinical skills teachers. Medical Teacher, 31(7), 634-641. 
ENQA. (2005). Standards and Guidelines for Quality Assurance in the European Higher Education Area. European Association for Quality Assurance in Higher Education, 41. doi:952-5539-05-9

Espinoza, Ó., \& González, L. E. (2013). Accreditation in higher education in Chile: results and consequences. Quality Assurance in Education, 21, 20-38.

Friz, M., Sanhueza, S., \& Figueroa, E. (2011). Concepciones de los estudiantes para profesor de matemáticas sobre las competencias profesionales implicadas en la ensenanza de la estadística. Revista Electronica de Investigacion Educativa, 13, 113-131.

Fry, H., Ketteridge, S., \& Marshall, S. (2008). A handbook for teaching and learning in higher education: Enhancing academic practice. London: Routledge.

Gambi, M. O., \& González, C. A. (2013). Endeudamiento estudiantil y acceso a la educación superior en Chile. Revista Española de Investigaciones Sociológicas, 141, 91-112.

Gargallo, B. (2008). Estilos de docencia y evaluación de los profesores universitarios y su influencia sobre los modos de aprender de sus estudiantes. Revista Española de Pedagogía, 66(241), 425-445.

Gargallo, B., Sánchez, F., Ros, C., Ferreras, A. (2010). Estilos docentes de los profesores universitarios. La percepción de los alumnos de los buenos profesores. Revista Iberoamericana de Educación, 51. Recuperado de http://www.rieoei.org/3236.htm

Glenn, D., Patel, F., Kutieleh, S., Robbins, J., Smigiel, H., \& Wilson, A. (2012). Perceptions of optimal conditions for teaching and learning: a case study from Flinders University. Higher Education Research \& Development. doi:10.1080/07294360.2011.555390

Haig, A., \& Dozier, M. (2003). BEME Guide no. 3: systematic searching for evidence in medical education-Part 2: constructing searches. Medical Teacher, 25(4), 463-84.

Hannah, J., Stewart, S., \& Thomas, M. (2011). Analysing lecturer practice: the role of orientations and goals. International Journal of Mathematical Education in Science and Technology. doi:10 $.1080 / 0020739 X .2011 .610008$

Hativa, N., Barak, R., \& Simhi, E. (2001). Exemplary University Teachers: Knowledge and Beliefs Regarding Effective Teaching Dimensions and Strategies. The Journal of Higher Education, 72, 699-729.

Hernández, H., Fernández, C., \& Baptista, P. (2010). Metodología de la investigación (5ª ed.). México D.F: Mc Graw Hill.

Jahangiri, L., McAndrew, M., Muzaffar, A., \& Mucciolo, T. W. (2013). Characteristics of effective clinical teachers identified by dental students: a qualitative study. European Journal of Dental Education, 17(1), 10-18.

Katz, J., \& Spence, R. (2009). Chilean universities in the transition to a market-driven policy regime. Higher Education Management and Policy, 21(2), 1-18.

Küster-Boluda, I., \& Vila López, N. (2012). El docente universitario y sus efectos en el estudiante. Estudios Sobre Educación, 23, 157-182.

Lee, A., Manathunga, C., \& Kandlbinder, P. (2010). Shaping a culture: oral histories of academic development in Australian universities. Higher Education Research \& Development. doi:10.1080/07294360903252128

Marín, M., Martinez, R., Troyanor, Y., \& Teruel, P. (2011). Student Perspectives on the University Professor Role. Social Behavior and Personality: An International Journal. doi:10.2224/ sbp.2011.39.4.491

Martínez, M. del M., García, B., \& Quintanal, J. (2006). El perfil del profesor universitario de calidad desde la perspectiva del alumnado. Educación XX1, 9(1), 183-198.

Marzano, R. J. (2010). On excellence in teaching. Bloomington: Solution Tree.

Moher, D., Liberati, A., Tetzlaff, J., \& Altman, D. G. (2009). Preferred reporting items for systematic reviews and meta-analyses: the PRISMA statement. Annals of Internal Medicine, 151(4), 264-269.

Monereo, C., \& Domínguez, C. (2014). La identidad docente de los profesores universitarios competentes. Educación XX1, 17(2), 83-104.

OECD. (2013). Quality assurance in higher education in Chile 2013. In Quality assurance in higher 
education in Chile. Paris: OECD Publishing.

Parpala, A., Lindblom-Ylänne, S., \& Rytkönen, H. (2011). Students' conceptions of good teaching in three different disciplines. Assessment \& Evaluation in Higher Education, 36(5), 549-563.

Pozo, C., Bretones, B., José Martos, M., \& Alonso, E. (2011). Evaluación de la actividad docente en el Espacio Europeo de Educación Superior: un estudio comparativo de indicadores de calidad en universidades europeas. Revista Española de Pedagogía, 69(248), 145-163.

PUCV. (2013, Mayo 19). Radiografía Primera Generación de Universitarios. La Tercera, p. 16.

Sánchez, J. E. (2013). La excelencia en el profesor universitario. Revista Española de Pedagogía, 71(254), 11-28.

Singh, J. (2013). Critical appraisal skills programme. Journal of Pharmacology and Pharmacotherapeutics, 4(1), 76-77.

Singh, S., Pai, D. R., Sinha, N. K., Kaur, A., Soe, H. H. K., \& Barua, A. (2013). Qualities of an effective teacher: what do medical teachers think? BMC Medical Education, 13(128), 1-7.

Torok, S. E., McMorris, R. F., \& Lin, W.-C. (2010). Is Humor an Appreciated Teaching Tool? Perceptions of Professors' Teaching Styles and Use of Humor. College Teaching, 52(1), 14-20.

Vajoczki, S., Savage, P., Martin, L., Borin, P., \& Kustra, E. D. H. (2011). Good Teachers, Scholarly Teachers and Teachers Engaged in Scholarship of Teaching and Learning: A Case Study from McMaster University, Hamilton, Canada. Canadian Journal for the Scholarship of Teaching and Learning, 2(1), 1-29.

Van Der Leeuw, J., Van Dijk, N., Van Etten-Jamaludin, F., \& Wieringa-De Waard, M. (2013). The attributes of the clinical trainer as a role model: A systematic review. Academic Medicine, 88(1), 26-34.

Young, S., \& Shaw, D. G. (2014). Profiles of Effective College and University Teachers. The Journal of Higher Education, 70, 670-686. 